\title{
Insights into effects of topical application of cis-urocanic acid on the skin microbiome and immune modulation.
}

VijayKumar Patra $^{1}$, Meghana Somlapura ${ }^{2}$, Mina Bashir ${ }^{3}$, and Peter Wolf ${ }^{1}$

\section{Introduction:}

$>$ Urocanic acid (UCA) is present locally in the stratum corneum of the skin in its trans isoform (transUCA)

Upon ultraviolet-radiation (UV-R) trans-UCA is isomerized to cis-UCA. ${ }^{1}$

cis-UCA is known to induce immune suppression via $5-\mathrm{HT}_{2} \mathrm{~A}$ receptor, serotonin signaling and various other pathways. ${ }^{2}$

> Since skin microbiome is established all over the surface of the skin, we investigated the effects of cis-UCA on the skin microbiome and antimicrobial peptides (AMPs) expression and further investigated the immune modulation using mouse models. ${ }^{3}$

\section{Results:}

1. PUVA, UVA and UV-B isomerizes trans-UCA to cis-UCA.
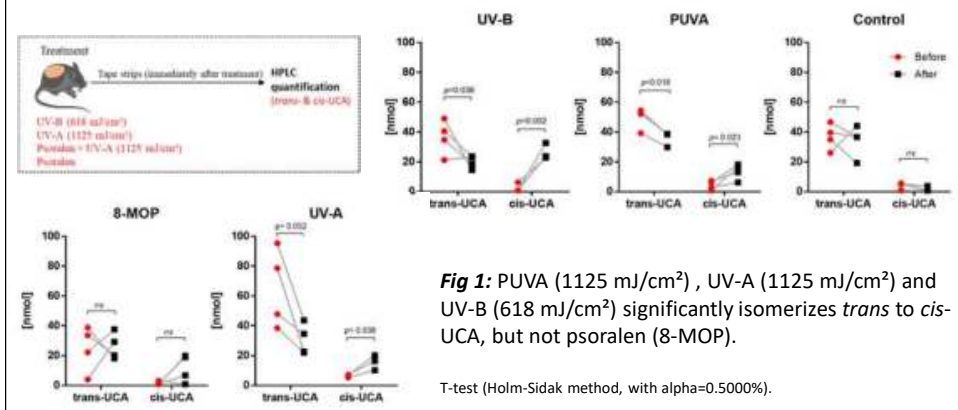

\section{2. cis-UCA modulates microbes on the skin.}

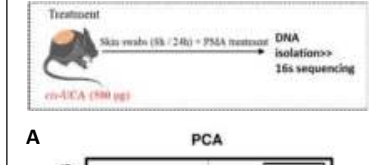
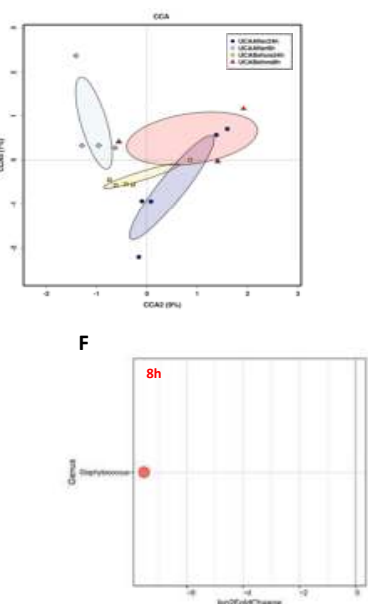
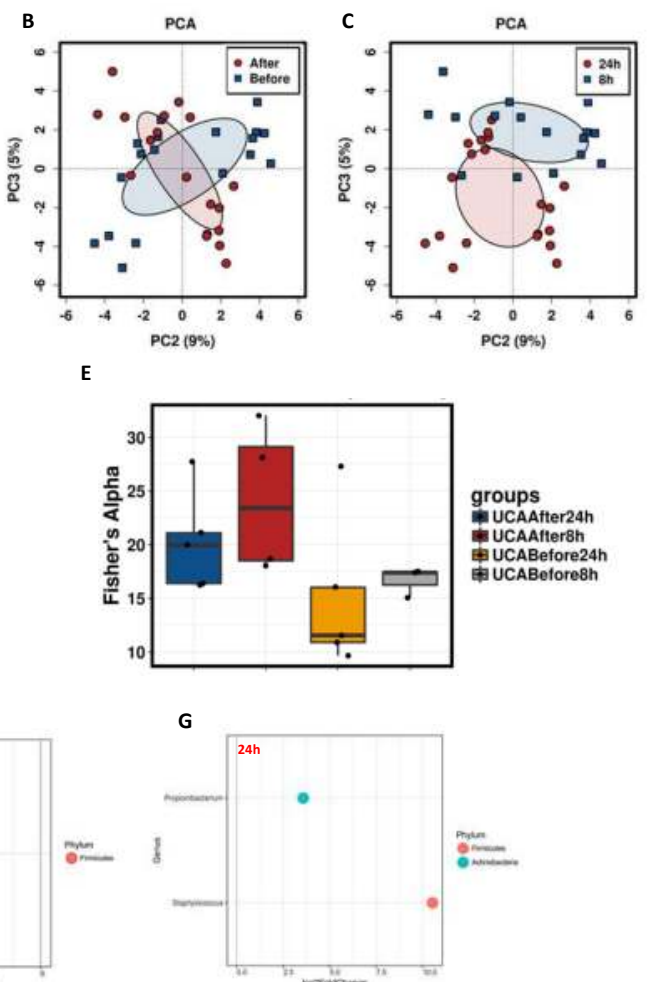

Fig 2: Principal component analysis (PCA) shows sample clustering between (A) controls and cis-UCA treated (B) before and after treatment and (C) timepoint; $8 \mathrm{~h}$ and $24 \mathrm{~h}$ after treatment. (D) Canonical correspondence analysis (CCA) shows clustering between cis-UCA treated groups. (E) Diversity analysis between cis-UCA treated groups. The prominent species affected by topical application of cis-UCA at (F) $8 \mathrm{~h}$ is Staphylococcus xylosus and (G) $24 \mathrm{~h}$ are Staphylococcus capitis/caprae and Propionibacterium acnes

\section{Results:}

3. cis-UCA modulates expression of antimicrobial peptides in the skin.

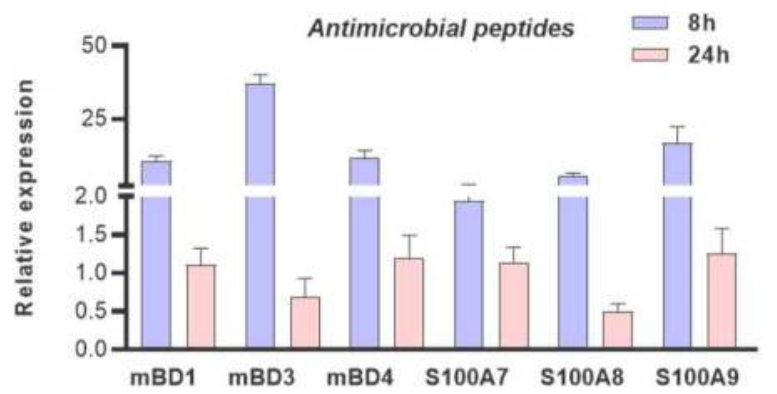

Fig 3 : AMPs gene signature significanlty differs at $8 \mathrm{~h}$ and $24 \mathrm{~h}$. Expression of AMPs is reduced at $24 \mathrm{~h}$ after cis-UCA $(500 \mu \mathrm{g})$, compared to $8 \mathrm{~h}$.

4. Disinfecting the skin results in reduced isomerization of trans- to cis- UCA.

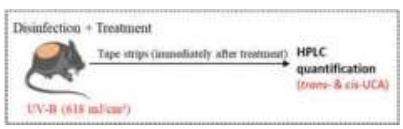

Fig 3 : Topical disinfection by chlorhexidine, followed by UVB treatment significantly reduces the isomerization of urocanic acid.

T-test (Holm-Sidak method, with alpha $=0.5000 \%$.

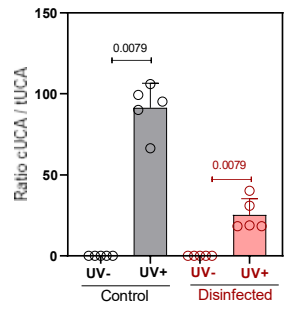

5. cis-UCA, PUVA and UV-B suppress immune reaction to contact allergen DNFB.

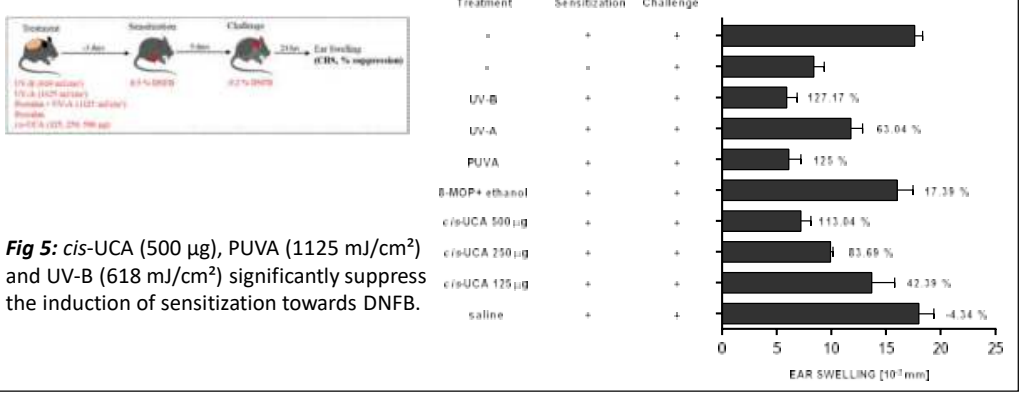

\section{Summary and Conclusion:}

PUVA, UV-A and UV-B significantly

increase the formation of cis-UCA.

Application of cis-UCA on the skin alters the microbial landscape and AMP gene expression of the skin.

$\checkmark$ Disinfecting the skin reduces the isomerization of urocanic acid.

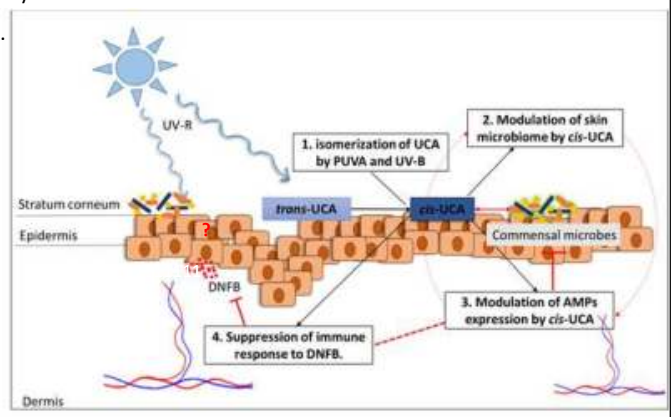

$\checkmark$ There is dose-dependent increase in immune suppression against the contact allergen DNFB, when the mice are pre-treated with cis-UCA .

$\checkmark$ cis-UCA may have a role in immune suppression through affecting skin microbiome and AMPs expression.

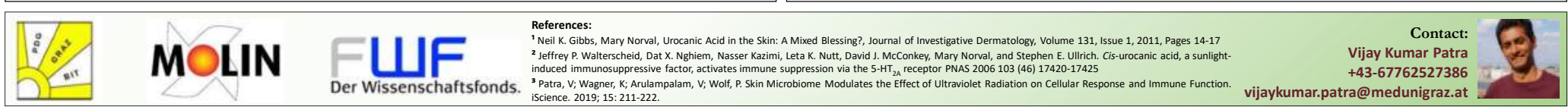

